\title{
MULTIOBJECTIVE APPROACH IN PLANS FOR TREATMENT OF CANCER BY RADIOTHERAPY
}

\author{
Thalita Monteiro Obal ${ }^{1,2^{*}}$, Neida Maria Patias Volpi ${ }^{1}$ \\ and Simone Aparecida Miloca ${ }^{1}$
}

Received November 21, 2011 / Accepted January 23, 2013

\begin{abstract}
Nowadays the technique of radiotherapy has been one of the main alternatives for the treatment of several types of cancer today. With technological development, especially in the case of 3D conformal radiotherapy, applications involving mathematical techniques and algorithms have been proposed to help the development a good treatment plan. This paper aims at present a model for multiobjective linear programming problem of dose intensity. The focus of the model is to determine the best dose distribution of radiation field, so that the dose delivered to the tumor to be prescribed and that affects the minimum the noble and healthy tissues. A test case of prostate cancer was used as an example of the numerical model and the Pareto-Optimal Frontier was generated using the method of weighted function.
\end{abstract}

Keywords: 3D conformal radiotherapy, multiobjective programming, method of weighted function.

\section{INTRODUCTION}

The radiotherapy technique is one of the most important alternative for the treatment of cancer nowadays. This type of treatment is based on blocking or destructing the cell division in the DNA molecules that make up the tumor and consisting irradiating the tumor to maximize the effect of radiation on the affected tissues, minimizing adverse impacts on other body tissues. To combat this evil, much has been invested in technology and research.

Most radiotherapy treatment centers in Brazil have modern equipments for radiation emission, the linear accelerators. The equipment has computer systems to support the decision that play a central role in allowing the manipulation of images and the simulation of the effects of a treatment regimen.

However, these computer systems can reach quite high costs of implementation and maintenance. Also it do not perform automatic optimization procedures, which is the responsibility of the

\footnotetext{
*Corresponding author

${ }^{1}$ Universidade Federal do Paraná, UFPR, Brazil.

${ }^{2}$ Universidade Tecnológica Federal do Paraná, UTFPR, Brazil.

E-mails: thalitaobal@utfpr.edu.br /neida@ufpr.br/smiloca@gmail.com
} 
planner's experience and intuition or by trial and error approach, can generate a solution away from optimal.

The plane of cancer treatment is performed individually but following the same methodology. The treatment plan is carefully developed, based on 3D computed tomography images of the patient, in conjunction with computerized dose calculations to determine the dose intensity pattern that will best conform to the tumor shape. There are three optimization problems relevant to be treated in a treatment plan.

(i) The geometrical problem;

(ii) The problem of dose intensity;

(iii) The problem of the blades opening.

The three problems are the central goal of providing the required dose to eliminate the tumor, with the lowest possible dose to healthy organs (called noble tissues), as well as other body tissues, called healthy tissues.

The first problem is to determine the optimal set of directions for each of the radiation beams (Figure 1).

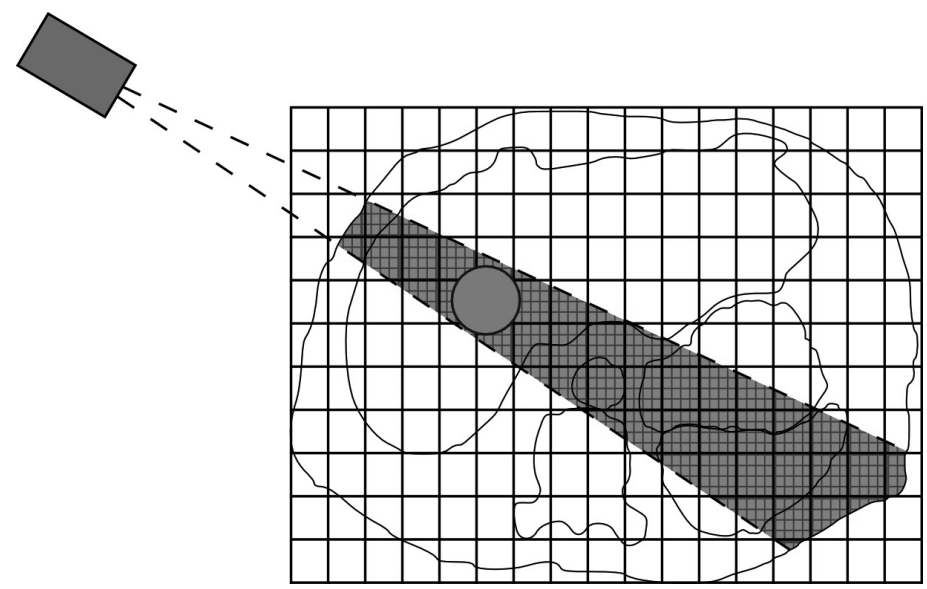

Figure 1 - Issuance of a radiation beam. (Source: Goldbarg, 2009).

In recent years, several strategies have been used in the search for solutions to this first problem. Goldbarg et al. (2009) propose that the problem of selecting the directions of the beams is done using a technique called variable isocenters. The multiobjective mathematical programming model is solved by Transgenetic Algorithm and also with a Multiobjective Genetic Algorithm with comparative purpose. Another approach to this problem has been presented by Araújo (2010). He proposes a generic model for the optimization of radiotherapy treatment plan, and he uses three metaheuristics called MOGA, MOSA and MOTS to seek its solutions. 
In Potrebko et al. (2007), an algorithm to optimize the angle of the radiation beam is proposed, based on minimizing the intersection the amount of the radiation beam in organs at risk. The algorithm was applied for optimization of coplanar beam arrangements regularly spaced in treatment of prostate cancer. The authors present a strong correlation between the minimization the amount of the intersection of the radiation beam in the organs at risk with high-dose.

The second problem is the dose intensity and aims at determining the best distribution dose for each radiation beam.

In this regard, Barboza et al. (2006) use the interior point method to solve the model introduced by Holder (2003). It incorporates elastic constraints that satisfy all constraints for treatment when the solution exists, or has the best solution out of specification, possible to according a weighting of the objective function. Another approach made to the model proposed in Holder (2003) is given by Viana (2010), taking into account the factors of correction for the heterogeneity in the composition of different types of tissue irradiated, based on proportions between their different linear attenuation coefficient.

Also based on the model presented by Holder (2003), Shao (2008) solves the problem of dose intensity through a variant of the method developed by Benson (1998), applying it to a clinical problem in 3D voxel using $5 \mathrm{~mm}$ and $3 \mathrm{~mm}$.

The third problem seeks to establish the best opening of the multileaf collimator (Figure 2) taking the shape of the tumor.

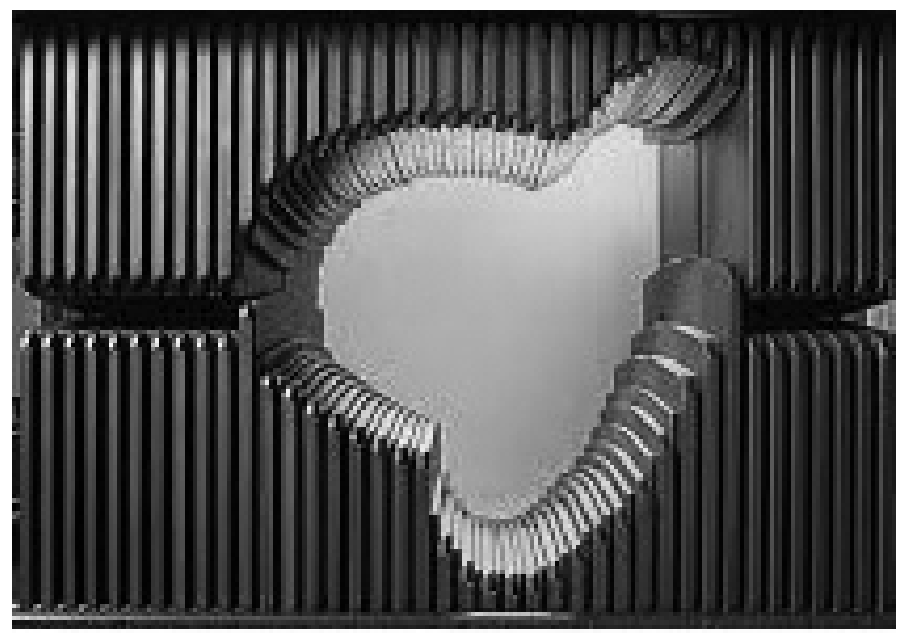

Figure 2 - Multileaf collimator. (Source: Holder, 2003).

This problem is treated mathematically by Cambazard (2009), and can be formulated as the decomposition of an integer matrix into a weighted sum of $0 / 1$ matrices (Figure 3 ), in which each row has the "consecutive ones property" and the state-of-the-art approach is based on constraint programming. 

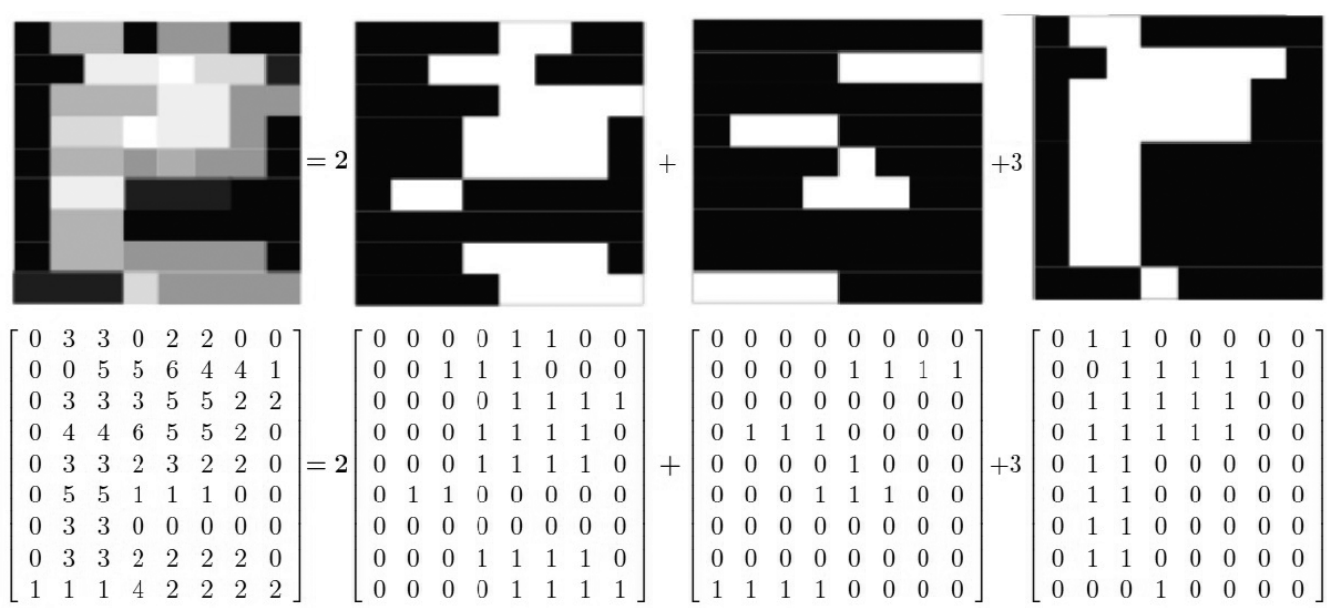

Figure 3 - The problem of the sequencing of the blades. (Source: Cambazard, 2009).

Developing a treatment planning is non-trivial. For example, a treatment planner might desire the tumor to receive no less than $80 \mathrm{~Gy}$. Similarly, the planner may hope that some other critical structures receive no more than $40 \mathrm{~Gy}$. So considering that the planner has many options, the use of tools that are capable of generating sets of optimal solutions regarding the impact areas affected by the radiation is helpful.

The problems involved in 3D conformal radiotherapy, from the point of mathematical view, are multiobjective large optimization NP-hard problems (Goldbarg, 2009). The three issues are treated independently in referred literature, because of the complexity of each, which does not exclude the possibility of treating them in an integrated way. Holder et al. (2008), discuss the difficulty of integrating the problems.

This paper proposes a methodology for a dose intensity problem in 3D conformal radiotherapy and a case study is presented as an example of aplication.

\section{FORMULATION OF THE PROBLEM OF DOSE INTENSITY}

Supposing the existence of a set of $k$ radiation beams previously defined, the problem is modeled considering a region of the human body obtained from a cut of tomographic image, as shown in Figure 4.

This region is represented by a grid of pixels. Each pixel is considered part of the healthy tissue, tumor, or noble.

The intention is to determine the amount of dose to be issued for each radiation beam, considering the dose absorption of different tissue, looking for the best configuration that allows the dose that reaches the tumor is sufficient to destroy it and that meets the dose limits in noble and healthy tissues. 


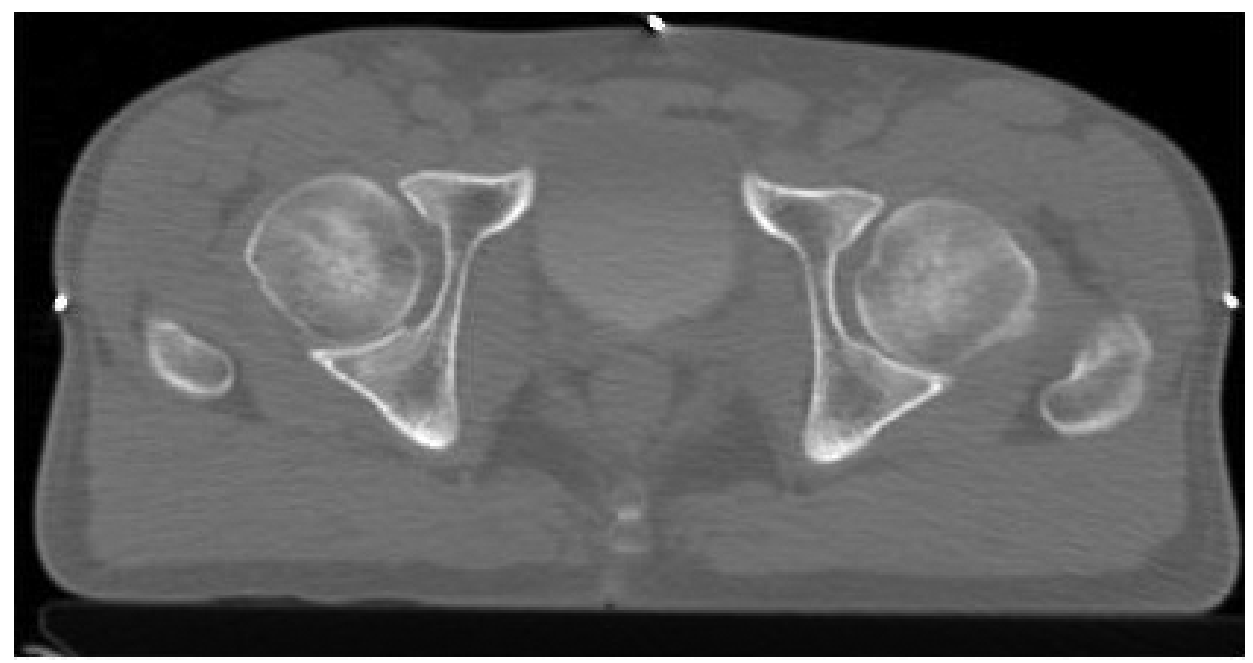

Figure 4 - Slice of tomographic image of the prostate region. (Source: Erasto Gaertner Hospital, Curitiba/PR.)

This problem is characterized by more than one objective, namely, the concern is not only radiating the tumor to maximize the effect of radiation on the affected tissues, but also having a concern as to minimize adverse impacts on other body tissues, so the model can be formulated as a multiobjective optimization model.

\subsection{Matrix Absorption Dose}

The dose that leaves from each radiation beam is not the same that will reach the tumor. There are several factors that reduce the dose, so that in each region of the body, or better in each pixel, there are different absorption dose. So, it is necessary to construct a matrix that will quantify the absorption dose per pixel, to each unit of radiation emitted by the beam.

Let $F^{k}$ be the matrix of the measure of the factors that influence the energy loss (by radiation beam $k$, in each pixel $(i, j))$.

One of the factors included in $F^{k}$, for example, is the PDP (Percentage depth dose), which can be measured experimentally. This factor measures the radiation that is received as a function of depth in the dose emitted, whose behavior can be visualized by mean of Figure 5.

Let $C^{k}$ the matrix that identifies the pixels hit by the beam $k$, so that:

$$
c_{i j}^{k}=\left\{\begin{array}{cc}
1 & \text { if the pixel }(i, j) \text { is affected by the radiation emitted by the field } k \\
0 & \text { otherwise }
\end{array}\right.
$$

To obtain a single matrix $A^{k}$ which considers all factors that influence the absorption of radiation at each pixel, is also necessary to consider the matrix of the factors of absorption due to the 


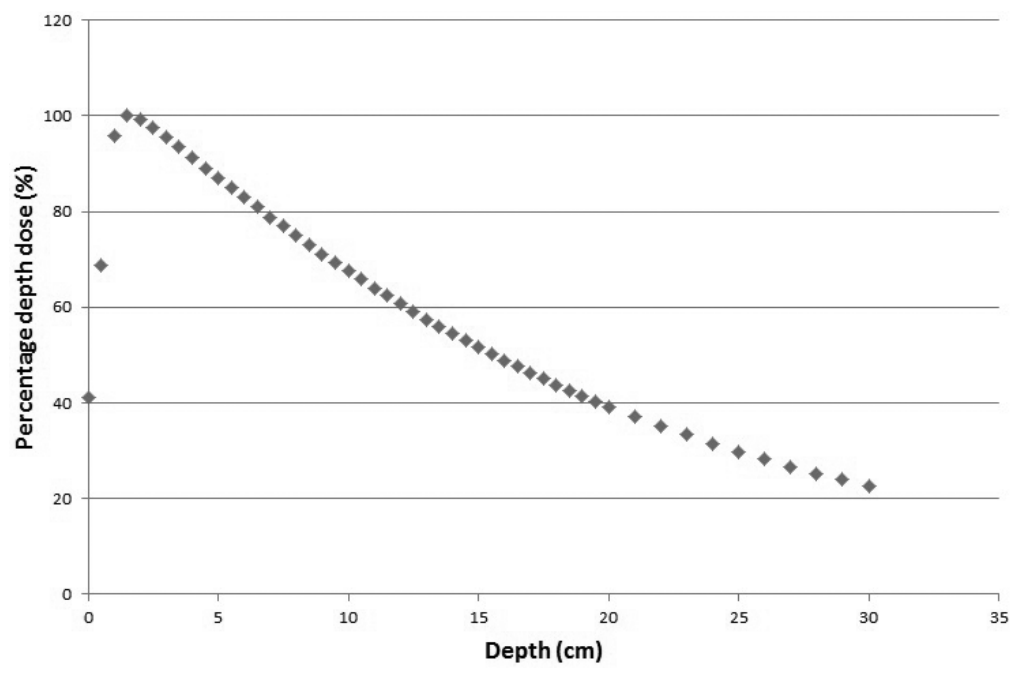

Figure 5 - Profile of attenuation of the radiation beam with respect to water depth.

heterogeneity in the composition of the irradiated tissue. One way to analyze this absorption is through the shades of gray in tomographic imaging, analyzed in the matrix $C T$.

The $c t_{i j}$ are values between zero and one, so that the darker the image, this value approaches zero, and the clearer, the value approaches one. Thus, regions of the body clearer in image, such as bone, for example, absorb more energy than the dark regions.

Thus, the matrix that considers all factors of energy absorption is given by:

$$
A^{k}=C T \odot F^{k} \odot C^{k}
$$

The symbol " $\odot$ " represents the multiplication point-to-point of the elements of the matrices.

Let $B_{n}, B_{S}$ and $B_{t}$ matrices that associate each pixel as noble, healthy or tumor, respectively.

$$
\begin{aligned}
& B_{n}=\left\{\begin{array}{lc}
1 & \text { if the pixel }(i, j) \text { is noble } \\
0 & \text { otherwise }
\end{array}\right. \\
& B_{s}=\left\{\begin{array}{lc}
1 & \text { if the pixel }(i, j) \text { is healthy } \\
0 & \text { otherwise }
\end{array}\right. \\
& B_{t}=\left\{\begin{array}{lc}
1 & \text { if the pixel }(i, j) \text { is tumor } \\
0 & \text { otherwise }
\end{array}\right.
\end{aligned}
$$

So:

$$
\begin{aligned}
& A_{n}^{k}=A^{k} \odot B_{n} \\
& A_{s}^{k}=A^{k} \odot B_{s} \\
& A_{t}^{k}=A^{k} \odot B_{t}
\end{aligned}
$$


are the matrices which represent the dose absorption in pixels noble, healthy and tumor, respectively, for each $k$ beam.

\subsection{Objective functions and constraints of the problem}

The objective is to determine the amount of $x_{k}$ dose to be issued for each $k$ beam of radiation, restricted to the dose limits for each type of tissue and considering the attenuation suffered by the dose emitted due to several factors. The unit of $x_{k}$ is the gray $(G y)$, which is a unit of absorbed dose, defined as the amount you deposit 1 joule $(J)$ of energy per kilogram $(\mathrm{kg})$ from the absorber mean.

The dose determination must be such that the issued dose that reaches to the pixels healthy and noble is the minimum possible and that the tumor dose is the closest to the prescribed by the doctor.

For this, in the model are considered deviations dose per pixels, allowing flexibility in the choice of dose. The matrices $\theta, \delta$ and $\epsilon$ represent the deviations of dose and are free variables.

$$
\begin{aligned}
& \theta=\theta^{+}-\theta^{-} \\
& \delta=\delta^{+}-\delta^{-} \\
& \epsilon=\epsilon^{+}-\epsilon^{-}
\end{aligned}
$$

The matrices $\theta^{+}, \delta^{+}$and $\epsilon^{+}$are matrices that represent the deviations of dose in excess in the pixels related to the noble, healthy and tumor, respectively. The matrices $\theta^{-}$and $\delta^{-}$represent the dose deviations below the upper limit of the dose of pixels of noble and healthy, respectively, and $\epsilon^{-}$is a matrix of deviations dose of tumor deficient in pixels.

The dose that reaches to the noble and healthy pixels must meet the upper dose $S_{n}$ and $S_{S}$, respectively. Then:

$$
\left\{\begin{array}{l}
\sum_{k=1}^{m} x_{k} A_{n}^{k} \leq S_{n} B_{n} \\
\sum_{k=1}^{m} x_{k} A_{s}^{k} \leq S_{S} B_{S}
\end{array}\right.
$$

where $m$ represents the number of radiation beams to be used.

Considering that for each pixel can be flexibility in the choice of the absorbed dose, the restrictions are thus rewritten:

$$
\left\{\begin{array}{l}
\sum_{k=1}^{m} x_{k} A_{n}^{k}=S_{n} B_{n}+\theta^{+}-\theta^{-} \\
\sum_{k=1}^{m} x_{k} A_{s}^{k}=S_{S} B_{s}+\delta^{+}-\delta^{-}
\end{array}\right.
$$

Moreover, $D$ is the constant that represents the dose prescribed by the doctor. As the pixels of tumor dose should be given $D$, another constraint in the model is in Eq. (14):

$$
\sum_{k=1}^{m} x_{k} A_{t}^{k}=D B_{t} \text {. }
$$


Also for the pixels of the tumor, it is considered an absorbed dose of flexibility, represented by the matrices $\epsilon^{+}$and $\epsilon^{-}$

$$
\sum_{k=1}^{m} x_{k} A_{t}^{k}=D B_{t}+\epsilon^{+}-\epsilon^{-}
$$

The model variables $x_{k},\left(\theta_{i j}^{+}\right),\left(\theta_{i j}^{-}\right),\left(\delta_{i j}^{+}\right),\left(\delta_{i j}^{-}\right),\left(\epsilon_{i j}^{+}\right)$and $\left(\epsilon_{i j}^{-}\right)$must all be non-negative.

The objective functions are shown in (16). Two objective functions of the model are to minimize the dose deviation over the pixels of noble and healthy, because they want to get the minimum dose in these pixels. The third and fourth objective are to minimize the dose matrices of deviations of excess and lack of tumor dose because the dose that reaches the tumor should be closest to the prescribed by the doctor.

$$
\left\{\begin{array}{l}
\text { Min } f\left(\theta^{+}\right)=\sum_{i=1}^{l} \sum_{j=1}^{c}\left(\theta_{i j}^{+}\right) \\
\text {Min } f\left(\delta^{+}\right)=\sum_{i=1}^{l} \sum_{j=1}^{c}\left(\delta_{i j}^{+}\right) \\
\text {Min } f\left(\epsilon^{+}\right)=\sum_{i=1}^{l} \sum_{j=1}^{c}\left(\epsilon_{i j}^{+}\right) \\
\text {Min } f\left(\epsilon^{-}\right)=\sum_{i=1}^{l} \sum_{j=1}^{c}\left(\epsilon_{i j}^{-}\right)
\end{array}\right.
$$

where $l$ and $c$ represent the number of rows and columns of the matrices $\theta^{+}, \delta^{+}, \epsilon^{+}$and $\epsilon^{-}$.

\subsection{Model for implementing the method of weighted function}

In the solution of multiobjective problems, two importants aspects are considered: the search for solutions and decision making. For the search of solutions, there is not an optimal solution with respect to all objectives, but rather a set of solutions called efficient solutions (or Pareto-optimal) in which no other solution that is best for all objectives. The image of the Pareto-optimal set is called the Pareto Front. As to the decision, the decision maker is responsible for choosing a particular efficient solution to consider the overall objectives of the problem (Goicoechea et al. (1982)).

The method used to obtain the Pareto front in the model presented in section 2.2 is the weighted function method, one of the classical methods of multiobjective optimization. This method consists in converting the original multiobjective problem into a scalar single-objective problem using different weights for each objective. To obtain Pareto-optimal solutions, we solve the problem iteratively considering different weight vectors (Deb (2009), Goicoechea et al. (1982)).

Thus, the objective functions are rewritten as shown below.

$$
\operatorname{Min}\left(\alpha \sum_{i=1}^{l} \sum_{j=1}^{c}\left(\theta_{i j}^{+}\right)+\beta \sum_{i=1}^{l} \sum_{j=1}^{c}\left(\delta_{i j}^{+}\right)+\gamma_{1} \sum_{i=1}^{l} \sum_{j=1}^{c}\left(\epsilon_{i j}^{-}\right)+\gamma_{2} \sum_{i=1}^{l} \sum_{j=1}^{c}\left(\epsilon_{i j}^{+}\right)\right)
$$


subject to

$$
\left\{\begin{array}{c}
\sum_{k=1}^{m} x_{k} A_{n}^{k}=S_{n} B_{n}+\theta^{+}-\theta^{-} \\
\sum_{k=1}^{m} x_{k} A_{s}^{k}=S_{s} B_{s}+\delta^{+}-\delta^{-} \\
\sum_{k=1}^{m} x_{k} A_{t}^{k}=D B_{t}+\epsilon^{+}-\epsilon^{-} \\
x_{k} \geq 0 \\
\left(\theta_{i j}^{+}\right),\left(\theta_{i j}^{-}\right),\left(\delta_{i j}^{+}\right),\left(\delta_{i j}^{-}\right),\left(\epsilon_{i j}^{+}\right),\left(\epsilon_{i j}^{-}\right) \geq 0
\end{array}\right.
$$

where $\alpha, \beta, \gamma_{1}$ and $\gamma_{2}$ represent the weights related to the respective dose deviation matrices $\theta^{+}, \delta^{+}, \epsilon^{-}$and $\epsilon^{+}$.

The solution generated by the model (17) is non-dominated if it satisfy the Theorem 1 (Deb (2009)).

Theorem 1. The solution to the problem presented in (17) is Pareto-optimal if the weights $\alpha, \beta, \gamma_{1}$ and $\gamma_{2}$ are positive for all objective functions.

\section{A CASE STUDY}

The test case for numerical example of the model refers to a prostate cancer, whose data were obtained in Erasto Gaertner hospital, Curitiba-PR. This choice is due to being located in a more simplified anatomical region. It was considered a treatment that uses four beams of radiation.

The upper limits for the dose considered noble and healthy tissues, respectively, were $S_{n}=45 G y$ and $S_{s}=50 G y$, and the dose to the tumor should reach $D=60 G y$, usual doses in the treatment of prostate cancer.

The reference image, shown in Figure 4, has been manipulated and exploited by the software MATLAB.

The image initially generated a matrix of order $220 \times 420$, determining 554404 variables in the model. Because of computational limitations, it was not possible to work with matrices generated from the original image. Holder et al. (2008) discuss this problem, noting that sometimes it is necessary to reduce the matrices so that problem can be solved. In this study, it was necessary to subject the image to a process of reducing the number of pixels, reducing it to $20 \%$ of the previous image.

The grayscale matrix $C T$, as well as matrices: $C_{k}$ regarding the incidence in pixel $i j$ for $k$ bundle, $B_{n}$ which indexes the pixels of tissue noble; $B_{s}$ which indexes the pixels of healthy tissue; $B_{t}$ which indexes the pixels of tumor tissue were obtained using the software MATLAB.

Were used tabulated data obtained in dosimetry (Mayle et al. (2007)) for matrix absorption dose $F_{k}$. In this case study, only the factor due to the type of treatment in relation to the distance factor, PDP, was used. Treatment was considered a constant focus-isocenter, held at 600-C linear accelerator with energy of $10 \mathrm{Mev}$ at a distance of $100 \mathrm{~cm}$ of the isocenter of the tumor.

With this information, we obtained the matrices $A_{n}^{k}, A_{s}^{k}$ and $A_{t}^{k}$ for $k=1,2,3,4$. 


\section{RESULTS AND DISCUSSION}

Using the methodology and model described in section 2.3 , the results are presented in Table 1 and were obtained through the toolbox linprog of MATLAB software.

The average objective function presented in Table 1, is equivalent to the average values of $f\left(\epsilon^{-}\right)$, $f\left(\epsilon^{+}\right), f\left(\theta^{+}\right)$and $f\left(\delta^{+}\right)$on the number of pixels corresponding tissue affected by radiation. For example:

$$
\bar{f}\left(\epsilon^{-}\right)=\frac{f\left(\epsilon^{-}\right)}{n_{t}}
$$

where $n_{t}$ is the number of tumor pixels hit by radiation.

The results presented in Table 1 show that when there is no concern to minimize the deviations of excess dose in healthy and noble pixels, a high dose is applied in some beams and a low-dose in others. This results a greatest dose than the dose limit imposed to the noble and healthy tissues. This solution does not represent a non-dominated solution, because it does not conform to Theorem 1, then is a solution that should be disregarded. The Figures 6 and 7 show the distribution of non-dominated solutions in objective space.

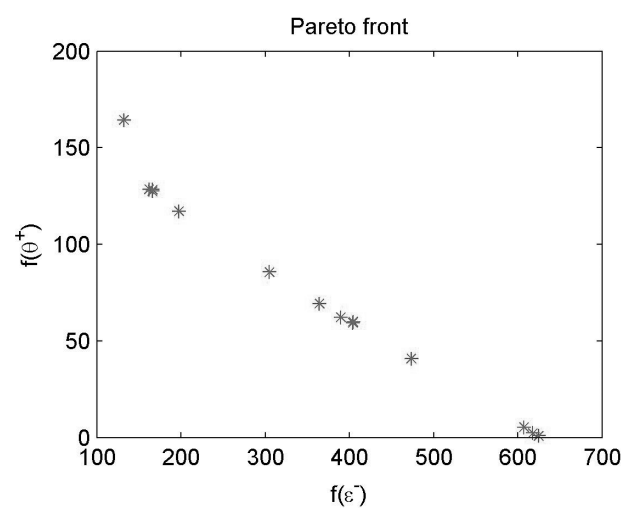

Figure 6 - Pareto front in relation to dose deviations $\epsilon^{-}$and $\theta^{+}$.

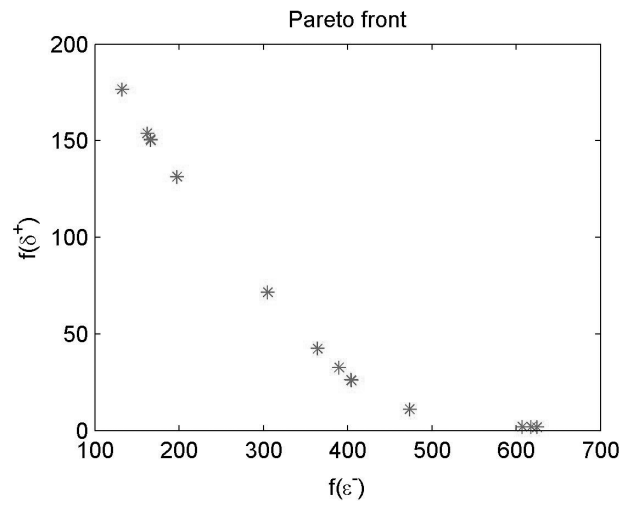

Figure 7 - Pareto front in relation to dose deviations $\epsilon^{-}$and $\delta^{+}$. 


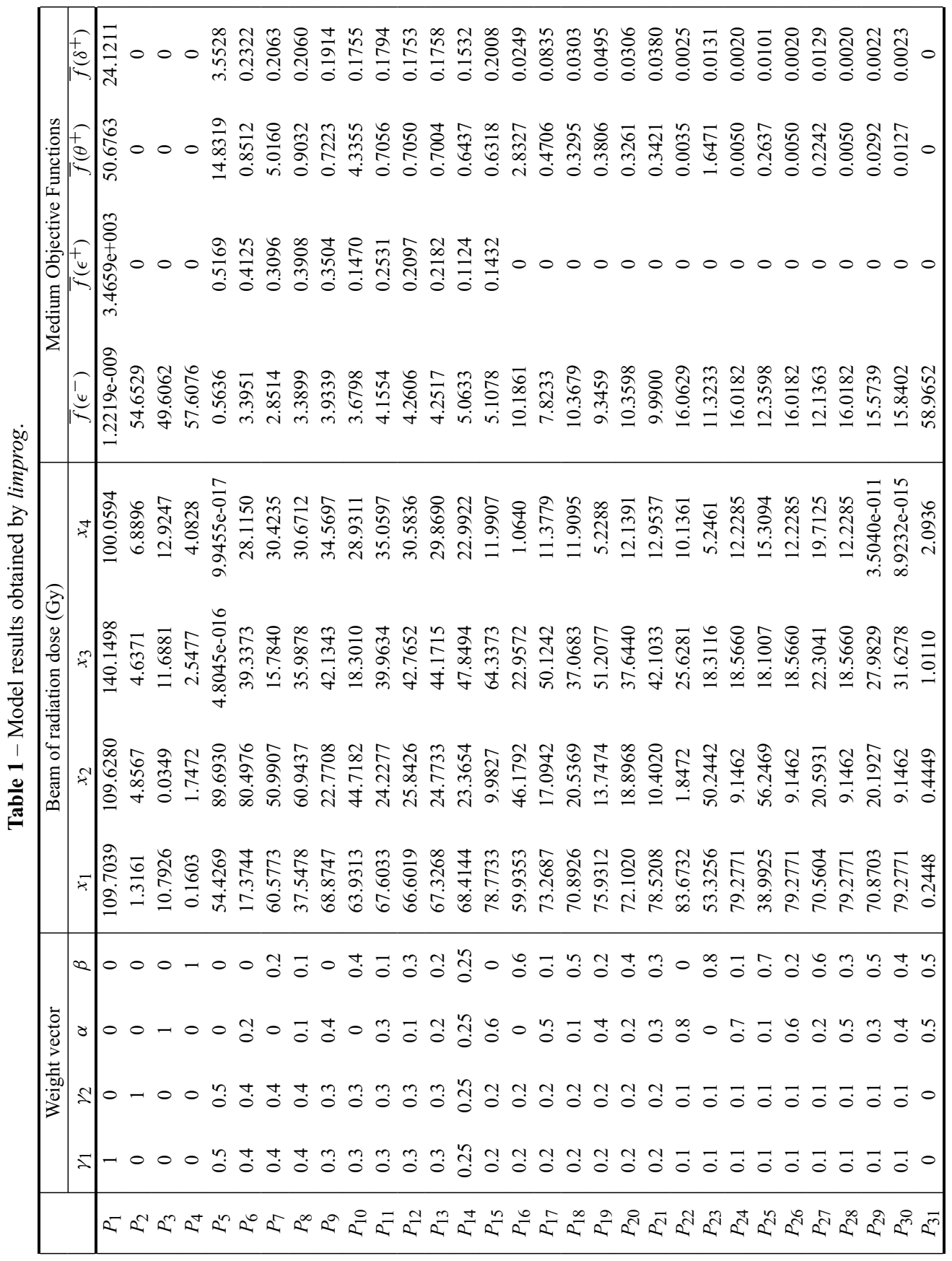


As for conflicting objectives, note that the closer the dose to be applied is the prescribed dose, or $f\left(\epsilon^{-}\right)$near zero, which is desirable for the tumor, greater is the deviation in the tissues noble and healthy, a result which is not desired for such tissue.

Although the methodology presented does not indicate a single optimal solution, but a set of compromise solutions, shown in Pareto frontier (Figures 6 and 7), the decision on which one is chosen as the final solution, it is the specialist decision maker, due to other clinical criteria. To help this choice, this paper suggested following criteria: the solution chosen is the one with the smallest euclidean distance in relation to the ideal point.

The ideal point is that whose coordinates are formed by the best solution for each objective (Deb (2009)). The Figure 8 shows the representation of the ideal point in relation to the solutions of the Pareto frontier, which has coordinates $\left(f\left(\epsilon^{-}\right), f\left(\theta^{+}\right), f\left(\delta^{+}\right)\right)=(3,3899 ; 0,0050 ; 0,0020)$.

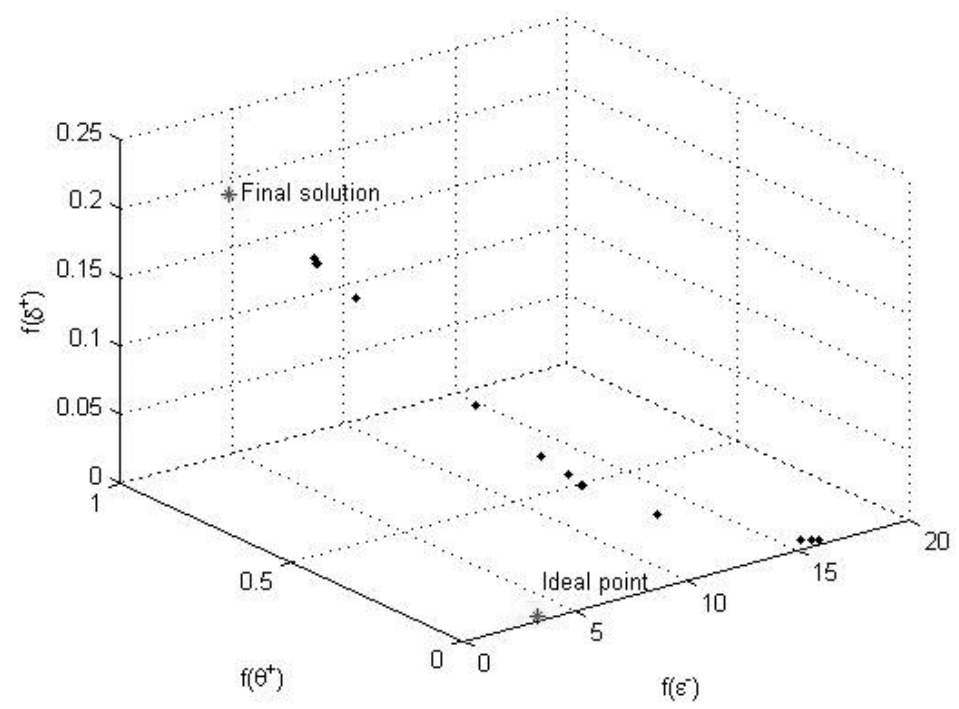

Figure 8 - Best solution in relation to ideal point.

According to the criterion used, the "best solution" was found was $f\left(\epsilon^{-}\right)=3.3899, f\left(\theta^{+}\right)=$ 0.9032 and $f\left(\delta^{+}\right)=0.2060$, with weights for each functions are $\gamma_{1}=0.4, \alpha=0.1$ and $\beta=0.1$, and the values of doses issued by each beams radiation is $x_{1}=37,54780, x_{2}=60,94370$, $x_{3}=35,98780$ and $x_{4}=30,6712$.

\section{CONCLUSIONS}

The problem of dose intensity was formulated as a multiobjective mathematical programming model, presented as a suitable and simple model. A study case was presented as numerical example and refers to a prostate cancer. The software MATLAB was used in the exploration of images, as well as for obtaining the solution, being efficient and fast in its execution. The weighted function method was used to search solutions, showing different configurations that 
allow comparisons between the results generated with different weight vectors. Pareto's front showed the importance of the decision maker in multiobjective problems. Observing that in conflicting situations it is necessary to penalize some factors to winning in others. It is proposed a criterion for the decision of choice.

\section{ACKNOWLEDGMENTS}

The authors would like to thank the team of radiotherapy of Erasto Gaertner Hospital, Curitiba - Paraná, with the number 2042 project approved by the Ethics Committee on Research. Thalita Monteiro Obal acknowledges the support of CAPES through scholarship REUNI.

\section{REFERENCES}

[1] Acosta R, Brick W, Hanna A, Holder A, Lara D, McQuilen G, Nevin D, Uhlig P \& SAlter B. 2008. Radiotherapy optimal design: An Academic Radiotherapy Treatement Design System, Matematics Faculty Research.

[2] ARAujo FS. 2010. Um estudo algorítmico para otimização do plano de tratamento em radioterapia conformal, Dissertação de Mestrado, Universidade Federal do Rio Grande do Norte, Natal.

[3] Barboza CB \& Oliveira AR. 2006. Planejamento do tratamento por radioterapia através de métodos de pontos interiores. Pesquisa Operacional, 26(1): 1-24.

[4] BENSON HP. 1998. Anouter approximation algorithm for generating all eficient extreme points in the out come set of a multiple objective linear programming problem. Journal of Global Optimization, 13: 1-24.

[5] Cambazard H, O’Mahony E \& O’Sullivan B. 2009. A Shortest Path-based Approach to the Multileaf Collimator Sequencing Problem. Integration of AI and OR Techniques in Constraint Programming of Combinatorial, 5574: 41-55.

[6] DEB K. 2009. Multi-objective optimization using evolutionary algorithms. Wiley.

[7] Goicoechea A, Hansen DR \& Duckstein L. 1982. Multiobjetive decision analysis with engineering and business applications. John Wiley \& Sons.

[8] Goldbarg MC. 2009. Algoritmo evolucionário para otimização do plano de tratamento em radioterapia conformal 3D. Pesquisa Operacional, 29(2): 239-267.

[9] HoldeR A. 2003. Designing radiotherapy plans with elastic constraints and interior point methods. Healt Care Management Science, 6: 5-16.

[10] Mayles P, Nahum A \& Rosenwald JC. 2007. Handbook of radiotherapy physics. Taylor \& Francis Group, LLC.

[11] Potrebko PS ET AL. 2007. A simple geometric algorithm to predict optimal starting gantry angles using equiangular-spaced beams for intensity modulated radiation therapy of prostate cancer. Medical Physics, 34: (10). 
[12] Shao L. 2008. Multiple Objective Linear Programming in Radiotherapy Treatment Planning. Thesis of doctory, Department of Engineering Science, School of Engineering, University of Auckland.

[13] ViANA RS. 2010. Programação Linear aplicada à criação de planejamentos otimizados em radioterapia. Dissertação de Mestrado, Programa de Pós-Graduação em Biometria, IB, UNESP. 